\title{
The Effect of Oxygen on the Electronic Structure of $\mathrm{MgB}_{2}$
}

\author{
J. C. Idrobo*, N. D. Browning ${ }^{* * * * * *}$ \\ *Dept. of Physics, University of California-Davis, Davis, CA 95616, USA \\ ** Dept. of Chemical Engineering and Material Science, University of California-Davis, Davis, CA \\ 95616, USA \\ *** National Center for Electron Microscopy, Lawrence Berkeley National Laboratory, Berkeley, CA \\ 94720
}

The discovery of superconductivity in $\mathrm{MgB}_{2}$, with a transition temperature of $\mathrm{T}_{\mathrm{c}}=39 \mathrm{~K}$ [1], has focused scientific attention towards an understanding of its superconducting properties. Superconductivity in $\mathrm{MgB}_{2}$ is driven by hole transport through the boron orbitals [2]. The presence of oxygen as segregates in the grain boundaries and precipitates in the bulk of polycrystalline materials [3] could have a large effect on the hole carrier concentration and therefore change the superconducting properties of $\mathrm{MgB}_{2}$. In this study, we elaborate the first experimental evidence that oxygen segregates in the bulk of $\mathrm{MgB}_{2}$ can have an effect on the hole carrier concentration.

The experiment was performed using Z-contrast imaging and electron energy loss spectroscopy (EELS) techniques. The Z-contrast images and EELS were obtained from the $200 \mathrm{kV} \mathrm{JEOL} 2010 \mathrm{~F}$ STEM at the University of Illinois at Chicago, which has an objective aperture $\sim 15 \mathrm{mrad}$, and an angular range of 0-52 mrad for EELS and 52-200 mrad for the annular dark field (ADF) detector. The spectra were acquired with an energy resolution of $1.2 \mathrm{eV}$ and energy dispersion of $0.2 \mathrm{eV}$.

Figure 1a shows a low-magnification image of a typical oxygen precipitate observed in these samples (dark spot, low contrast region). The size of the grain is about $2 \mu \mathrm{m}$. Figure $1 \mathrm{~b}$ shows the same region magnified. Here, the hexagonal shape of the precipitate can be distinguished, with a diameter of about $60 \mathrm{~nm}$. EEL spectra of the boron K-edge and oxygen K-edge were taken from the precipitate and the region around it (which also contains oxygen). Both spectra were background subtracted and corrected for multiple-scattering contributions. Figure $2 \mathrm{a}$ shows the boron K-edge spectrum for the precipitate (i), the region around the precipitate (ii) and a spectrum obtained for pure $\mathrm{MgB}_{2}$ (iii) displayed for comparison. The fine structure of spectrum ii shows clear differences from the precipitate and the pure $\mathrm{MgB}_{2}$. The pre-peak intensity (labeled a) in spectrum ii shows a sharper feature than the precipitate and the pure $\mathrm{MgB}_{2}$ spectra with a decrease in its intensity at 191 $\mathrm{eV}$. The peaks (labeled $\mathrm{b}$ and $\mathrm{c}$ ) are slightly higher and more pronounced for spectrum ii than the precipitate spectra and both are higher than the pure $\mathrm{MgB}_{2}$ spectrum. The oxygen spectra (figure 2b) for the two regions look completely different, and this can be taken as evidence of two different phases.

Although the atomic ratios calculated from the two different regions do not differ by more than 5\%, the change of intensity shown in figure $1 \mathrm{~b}$ with the differences shown in the oxygen K-edge spectra (figure 2b) indicates the presence of a different phase. More importantly, the region around the precipitate shows a drastic change in the boron fine structure, sharpness in the pre-peak and at 195 $\mathrm{eV}$ of the boron K-edge (figure 2a). This pre-peak has been the focus of diverse studies [4], and an increase in its intensity is associated with a better performance of $\mathrm{MgB}_{2}$ as a superconductor with a higher critical temperature. Work is underway to evaluate the origin of the change in the pre-peak shape and its effect on superconducting properties [5]. 


\section{References:}

[1] Nagamatsu J., Nakawa N. et al., Nature (London) 410, (2001) 63.

[2] Hirsch J. E., cond-mat/0102115, (2001)

[3] Klie R. F., Idrobo J. C., and Browning N. D. et al. Applied Physics Letters 80 (2002) 21.

[4] Klie R. F. et al, cond-mat/0211295, (2002). Submitted to Phys. Rev. B

[5] This work was sponsored by DOE under grant number DE-FG02-96ER45610.
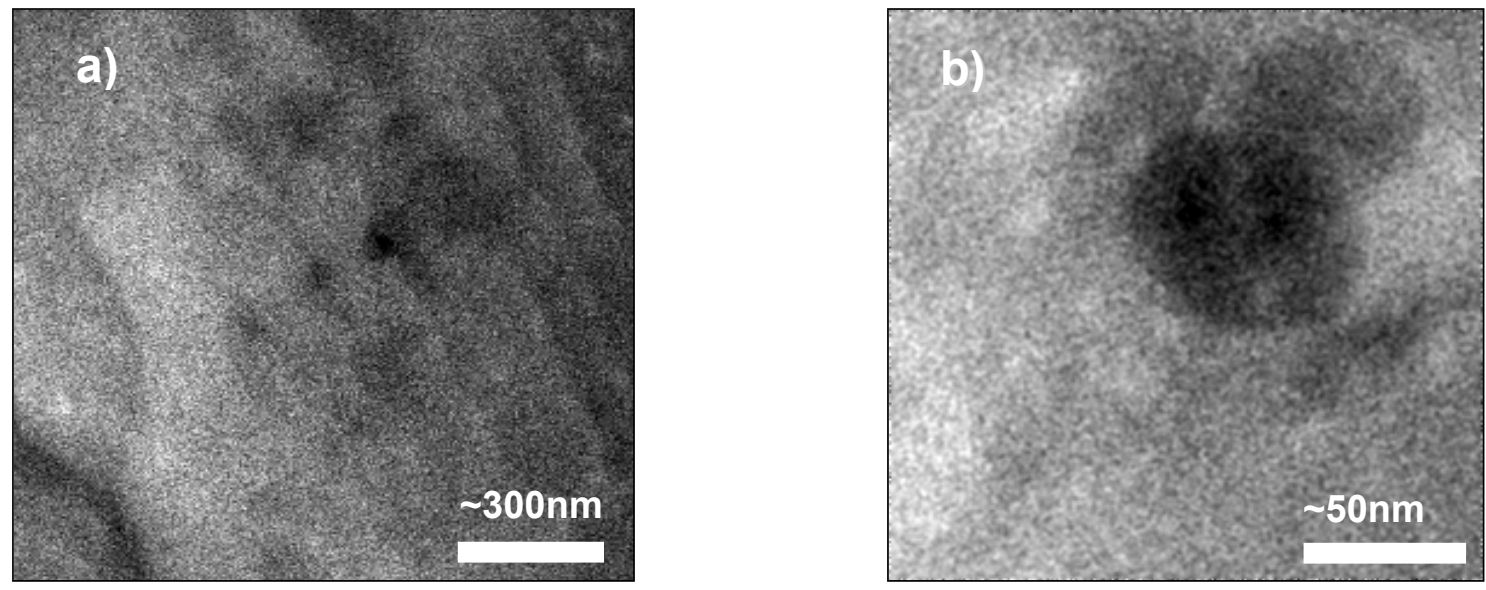

Figure 1. (a) Low magnification of polycrystalline $\mathrm{MgB}_{2}$. (b) High magnification of figure 1a, the change of contrast in the images indicates the differences phases in the material, bulk and precipitate.

a)

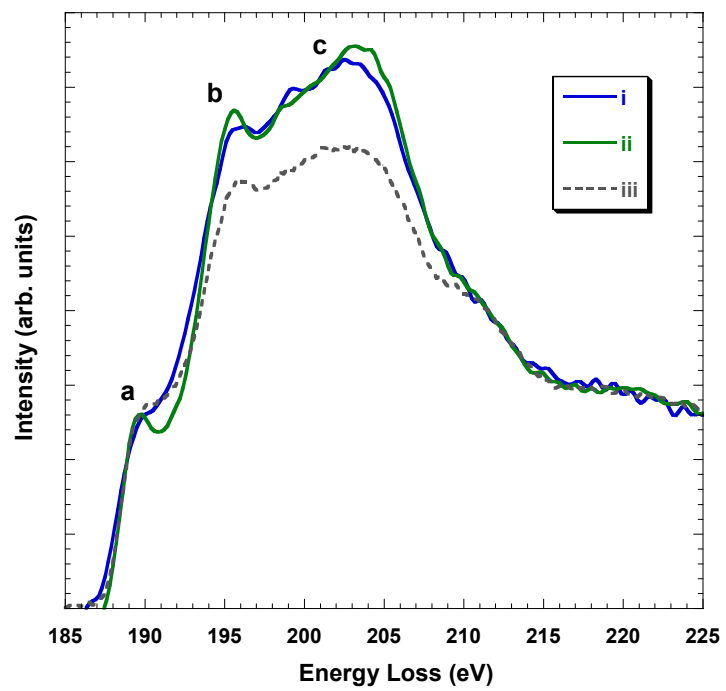

b)

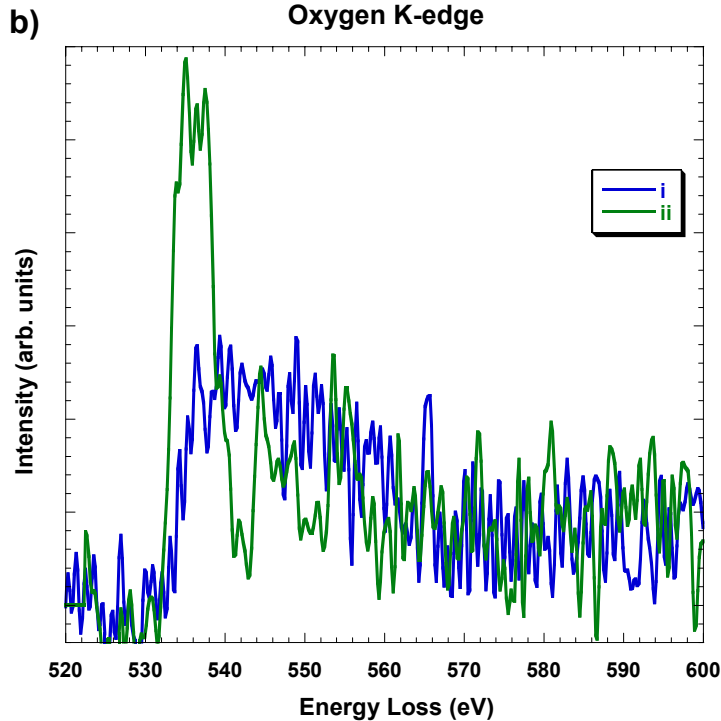

Figure 2. (a) Boron K-edge spectra, where differences can be observed in the fine structure. The changes of shape between the spectra in the pre-peak marked as $a$ and peak marked as $b$ are due to oxygen segregation in the bulk. (b) Oxygen K-edge spectra. The difference in the spectra for the two regions shows that oxygen has precipitated in $\mathrm{MgB}_{2}$ forming two different phases. 